INTERNATIONAL DESIGN CONFERENCE - DESIGN 2018

https://doi.org/10.21278/idc.2018.0215

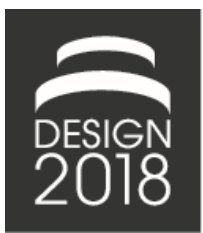

\title{
DEVELOPMENT OF A CATALOGUE SUPPORTING IDEA GENERATION FOR INTERNET OF THINGS USE CASES
}

\author{
J. Wilberg, K. Lau, T. Nützel, C. Hollauer and M. Omer
}

\begin{abstract}
Offering connected products that are part of the Internet of Things (IoT) enables new product functionalities and services, which creates additional revenues. Despite these new possibilities, engineering companies struggle to identify IoT use cases. Available publications mention many different use cases, but a structured overview is missing. This paper thus introduces a structured catalogue with 245 use cases. The catalogue provides a starting point to derive use cases based on analogy building. Initial case studies indicate that the catalogue assists in identifying new ideas for use cases.
\end{abstract}

Keywords: internet of things (IoT), big data analysis, design methods

\section{Introduction}

Digitalization is progressing at a fast pace and is currently a central topic for companies (Porter and Heppelmann, 2015). However, the digital transformation has multiple facets: automation, digital customer interaction, digital data, and connectivity (Bloching et al., 2015). Looking at the products of mechanical engineering companies reveals that more and more companies are developing and selling connected products. Such products do not only have physical parts, sensors, and other mechatronic elements, but also hold the ability to connect to the internet. Research and industry refer to such connected products as the Internet of Things (IoT) (Xia et al., 2012). Forecasts predict that the number of connected products in 2020 will be between 25-50 billion devices (Ellen MacArthur Foundation, 2016). These connected products enable companies to develop novel product and service concepts, which provide customers and users with personalized and tailored solutions (Erwin et al., 2015; Henke et al., 2016). The increasing number of connected products has already had a disruptive effect in many industries. Thus, product development and design processes in engineering companies are directly impacted because novel opportunities require new approaches (O’Halloran et al., 2015).

The key enabler for novel products and services is not just the possibility to connect products to the internet, but particularly the ability of connected products to transmit use phase data back to the manufacturer (van der Vegte, 2016). Use phase data includes all data "that is generated during the use phase by the product itself (e.g., by sensors or microprocessors) or by related services (e.g., Apps, maintenance reports, or repair reports)" (Wilberg et al., 2017b). Thus, access to use phase data enables companies to obtain novel insights about product usage and customer preferences. Depending on the complexity of the product (e.g., number of sensors) and the number of connected products in the field, large amounts of data are created. A new aircraft engine of Pratt \& Whitney has, for instance, more than 5000 sensors which create up to $10 \mathrm{~GB}$ of use phase data every second (Rapolu, 2016). 
This example should make it clear that merely having a warehouse full of data does not automatically create value (LaValle et al., 2011). Data should not be the starting point because companies should start with an idea of what they want to do with the data (LaValle et al., 2011). The key challenge for companies is to identify suitable use cases which create value (Davenport, 2014; Wirth and Wirth, 2017). A use case in the context of this paper is the procedure that describes how selected use phase data is processed in order to provide extra value for defined stakeholders (e.g., automated bill creation, theft protection, reduction of over-engineering, or predictive maintenance). Due to the broad range of possible use cases, companies need to explore the different options and select suitable ones. However, this is exactly where additional support is needed because companies inexperienced with selling connected products struggle to find use cases that add value to their product, service, or operations.

This paper therefore introduces a use case catalogue for mechanical engineering companies which are already offering or planning to offer connected technical products with related services. The developed use case catalogue uses analogy building in order to provide inspiration when searching for suitable use cases. The catalogue currently contains 245 different use cases from literature and case studies, which are clustered on three levels to enable companies to explore the wide range of options. The objective of the catalogue is not to provide a ready-to-implement use case, but rather to provide structured example use cases from other companies and industries which provide inspiration and serve as a starting point for the ideation of use cases that provide value for the user's company or customers. Thus, the underlying assumption is that a user benefits from reading about example use cases (e.g., self-learning thermostats sold by Nest) and is able to adapt this use case to the company's products (e.g., washing machines).

\section{Theoretical background}

This chapter provides a brief overview of the field of use phase data and the related use cases for IoT. The references were identified by defining specific combinations of keywords (e.g., IoT, product development, or product design). The goal was to analyse the range of possible use cases and the additional challenges that companies face when turning data into value. In order to identify relevant literature, different online databases such as EBSCO, ScienceDirect, IEEE Explore and GoogleScholar were searched. However, it also became clear that many reports about IoT and monetarizing data originates from consulting companies (e.g., McKinsey) or industrial associations (e.g., BITKOM).

\subsection{Internet of Things (IoT) and data analytics}

The advancement of technical products, which are nowadays often equipped with a certain intelligence and connectivity, has an extensive impact on companies and competition (Porter and Heppelmann, 2015). The term IoT is often used for such products, and means "networked interconnection of everyday objects, which are often equipped with ubiquitous intelligence" (Xia et al., 2012). IoT is a combination of the physical world (parts, sensors, and connectivity) and the digital world (connectivity, analytics, and digital service) (Fleisch et al., 2017). The connectivity element is thus the link between both worlds. Offering connected products allows for the integration of new features and functions, which leads to new business opportunities. In addition, collecting use phase data enables companies to gain in-depth insights into customer preferences and product usage (Erwin et al., 2015). Overall, connected products enable traditional engineering companies to provide additional value to internal stakeholders (e.g., product development or customer service) and external ones (e.g., users or retailers) (Jenkins, 2016).

In the past, companies in the consumer sector were a main producer of data, but in the last few years, the IoT trend has also turned the traditional manufacturing industry into a major producer of data because cars, aircraft engines or and elevators now produce use phase data (Bloching et al., 2015). Accessing and generating use phase data is one challenge that companies overcome more easily because of advances in IT. Due to size of the data however, the challenge is to process and analyse it (Bloching et al., 2015). In the recent years the term Big Data was often used to describe approaches and data analytics tools that allow for the handling of large data sets that, for instance, stem from IoT products. Literature often uses the terms volume, velocity, variety, value, and veracity (5 Vs) to characterize Big Data (Demchenko et al., 2014). Big Data tools play a key role in transforming use phase data stemming from connected products into value. 


\subsection{Examples for use cases using data analytics}

The previous section highlighted the close relation between the IoT trend and data analytics. In the past, companies like Amazon or Netflix overruled their competitors because data analytics became part of their business model (Muhtaroğlu et al., 2013). However, in the last few years departments which are less accustomed to using data, such as product development or logistics, have also started to incorporate data analytics (Dutta and Bose, 2015). It is important to mention that use cases depend not only on the department, but also on the industry sector (Erwin et al., 2015). The interesting aspect is that use phase data related use cases are possible during the entire product lifecycle, starting with conceptualisation and ending with the disposal of the product (Porter and Heppelmann, 2014).

Looking at mechanical engineering companies, research shows that companies from industry sectors like the automotive, aerospace, or industrial equipment sectors especially take advantage of use phase data (van der Vegte, 2016). More complex and expensive products in particular seem to be suitable for new data-based use cases. Companies can gain new insights into product usage, which allows for the segmentation of customers, provision of new services, or offering of additional product functions (Porter and Heppelmann, 2014). Looking at mechanical engineering companies, many use cases involve the improvement or enhancement of the products' maintenance (van der Vegte, 2016). In the context of maintenance, a frequently discussed use case is predictive maintenance (Bange et al., 2015). Additionally to this most common field of use cases, engineering companies state the use phase data can also support idea generation, requirements definition, the reduction of over-engineering, the detection of patterns in product failure, or the shortening of product testing (Wilberg et al., 2017a). Overall, use phase data makes new use cases in product development possible because such data provides novel insights concerning actual product usage (Porter and Heppelmann, 2014).

Looking at use cases from a customer perspective, use phase data can help to enhance performance (e.g., product flexibility), reduce costs (e.g., process costs), increase energy and resource efficiency (e.g., emission reduction), or avoid risks and increase security (e.g., quality management) (Kaeser, 2017).

\subsection{Barriers for extracting value from use phase data}

The connectivity of products enables many different use cases throughout the entire product life cycle. Nevertheless, these possibilities come at a price because implementing data-driven use cases requires changes not only from a technical perspective, but also from an organizational and strategic one. Selling connected products often entails that, for instance, services are added, which requires a new way or process to interact with the customer (Porter and Heppelmann, 2014). From a meta perspective, barriers arise mainly from three areas: technology, organization, and people (Alharthi et al., 2017).

Understanding the specific barriers is important in order to enable companies to overcome them. The access to use phase data is the key enabler. However, companies need to understand that just developing connected products does not per se confer any benefit for internal and external stakeholders (Fleisch et al., 2017). It is important that the physical part of the product and the digital part are aligned. A common mistake is that companies start with the collection of data before even knowing what they want to use it for (Barton and Court, 2012). The risk is that companies will collect a huge amount of use phase data without creating any benefit, but only additional cost and frustration (LaValle et al., 2011).

Another key challenge is that companies struggle to understand how connectivity could enhance their current product and business (O'Halloran et al., 2015). Furthermore, companies state that they are unsure about the return of investment when deciding to connect products and perform data analytics. Mechanical engineering companies especially, with pure physical products, have to learn how to integrate these new opportunities into their business (Capgemini and EMC, 2015). Survey results indicate that departments like marketing or sales use Big Data quite a lot already, but the results also highlight that the application of Big Data in R\&D will increase in the next years (Bange et al., 2015).

\subsection{The importance of a use phase data strategy}

Various researchers agree that the starting point for data driven use cases should not be the data itself (Barton and Court, 2012; Davenport, 2014). Often, companies do not struggle to collect data because connectivity makes use phase data easily accessible. However, commons mistake is to just instruct the 
employees to turn data into value without a plan or the belief that hiring skilled data analytics experts leads automatically to new insights (Fitzgerald et al., 2014).

Starting with data instead of proposed use cases or business opportunities bears the risk that companies will get lost in the resulting complexity and confusion because the objectives are not clear (LaValle et al., 2011). A major source of problems is the way that companies approach projects that turn data into value (Wirth and Wirth, 2017). Therefore, one of the most important steps is to derive a suitable and comprehensive data strategy (Barton and Court, 2012; Davenport, 2014). A clear use phase strategy should define which use cases a company plans to implement and which stakeholders should benefit from the use cases (Wilberg et al., 2017b). The process that identifies use cases and derives a strategy should be implemented in a structured way in order to ensure that all relevant objectives, needs, and stakeholders are considered (Wirth and Wirth, 2017). Strategy development should start with defining a vision and the overall objectives of the use phase data strategy (Wilberg et al., 2017b). Afterwards, an analysis phase follows that focuses on identifying the available use phase data as well as relevant internal and external stakeholders. A subsequent concept phase focuses on identifying, detailing, evaluating, and selecting suitable use cases, which is the foundation for deriving a use phase data strategy.

It is crucial to integrate the different stakeholders right from the beginning of the strategy development process to ensure that their diverse interests will be respected (Wirth and Wirth, 2017). Bringing together the different perspectives is crucial to avoid stakeholders refusing to use data for their decision making later on (Berman and Bell, 2011). Besides the more organizational challenges, defining and selecting use cases is the central task when developing a use phase data strategy (Wirth and Wirth, 2017). It is important that the use cases do not overburden the company and therefore it is good to focus on achievable use cases that fit the respective experience and skills (Barton and Court, 2012).

\section{Research gap and methodology}

\subsection{Research gap}

The previous chapter discussed the effect of digitalization, which creates a dynamic environment which engineering companies need to deal with. It became clear that IoT and Big Data are key enablers for the digital transformation that many companies are currently undergoing (Bloching et al., 2015). Connected products transmit use phase data that enables companies to improve products and services, which positively influences innovation and business success (Kusiak and Smith, 2007). However, the previous discussion also showed that these novel opportunities introduce new organizational or technical challenges. Mechanical engineering companies, like other companies that are working in a non-digital industry, require additional support to turn use phase data into value (Fleisch et al., 2017).

An important step for companies to master these new challenges is to develop a vison and strategy that describes how they want to use data to deliver extra value for internal and external stakeholders (LaValle et al., 2011; Wilberg et al., 2017b). Identification and selection of use cases is thus a critical task during the development of a use phase data strategy. However, use case identification is a twoedged sword. On the one side, a broad range of possible use cases exists. On the other side, companies struggle to identify suitable use cases due to the sheer number of possibilities and their missing experience. Selecting a use case has an extensive impact on the steps required to derive a strategy and implement the use case (Wirth and Wirth, 2017). In literature, different publications exist that provide a list of possible use cases for connected products (c.f. Chui et al., 2010; Dinter et al., 2015; Porter and Heppelmann, 2015). These publications provide limited help however for companies aiming to identify suitable use cases because they often just list a large number of use cases without always providing relevant details (e.g., required data or benefit). Furthermore, none of the available collections suggest a structured description of use cases, which would be helpful to better navigate through the different options. In contrast, publications exist that discuss certain use cases on a very detailed level (c.f. Carnero, 2005). An important research task to support companies in deriving a sound use phase data strategy is to cluster the motives for collecting data and the intended value of the use cases (van der Vegte, 2016). 


\subsection{Research objectives and methodology}

At the moment, literature only provides a very fragmented perspective on use cases for connected products and services. Companies that are not rooted in a digital industry, such as mechanical engineering companies, are especially in need of additional support to identify use cases that provide additional value and to derive a value adding use phase data strategy. The identification of use cases is the most critical task during strategy development. Exploring possible use cases is currently difficult because a comprehensive picture is missing. Companies that are less familiar with data-driven use cases can easily lose track of all the possible options. Some authors already address this by suggesting different clusters for use cases. Henke et al. (2016) mentions four use case categories: optimization of operations, predictive analysis, strategic optimization, and personalization. Another possibility is to divide use cases into operations, customer experience, and strategy (Barton and Court, 2012). From a customers' perspective, use cases either enhance performance, reduce cost, increase resource efficiency, or avoid risk (Kaeser, 2017). Nevertheless, the clusters mentioned above divide use cases on a very high-level. Each of the three or four clusters still contains a broad range of diverse use cases and therefore the authors believe that companies would benefit from a more detailed structure for use case categories.

Thus, the research objective is to provide additional support for companies during the ideation phase for use cases. The objective is to derive a comprehensive use case catalogue that makes the broad range of possible use cases visible. In addition, the objective is to support creative thinking during the search for suitable use cases by providing exemplary use cases from other companies. Examples from other research fields with many different options to choose from like business model development (Gassmann et al., 2014), PSS development (Schmidt et al., 2015), or bionics (Deldin and Schuknecht, 2014) show that such catalogues help the user to navigate through different options and select suitable ones.

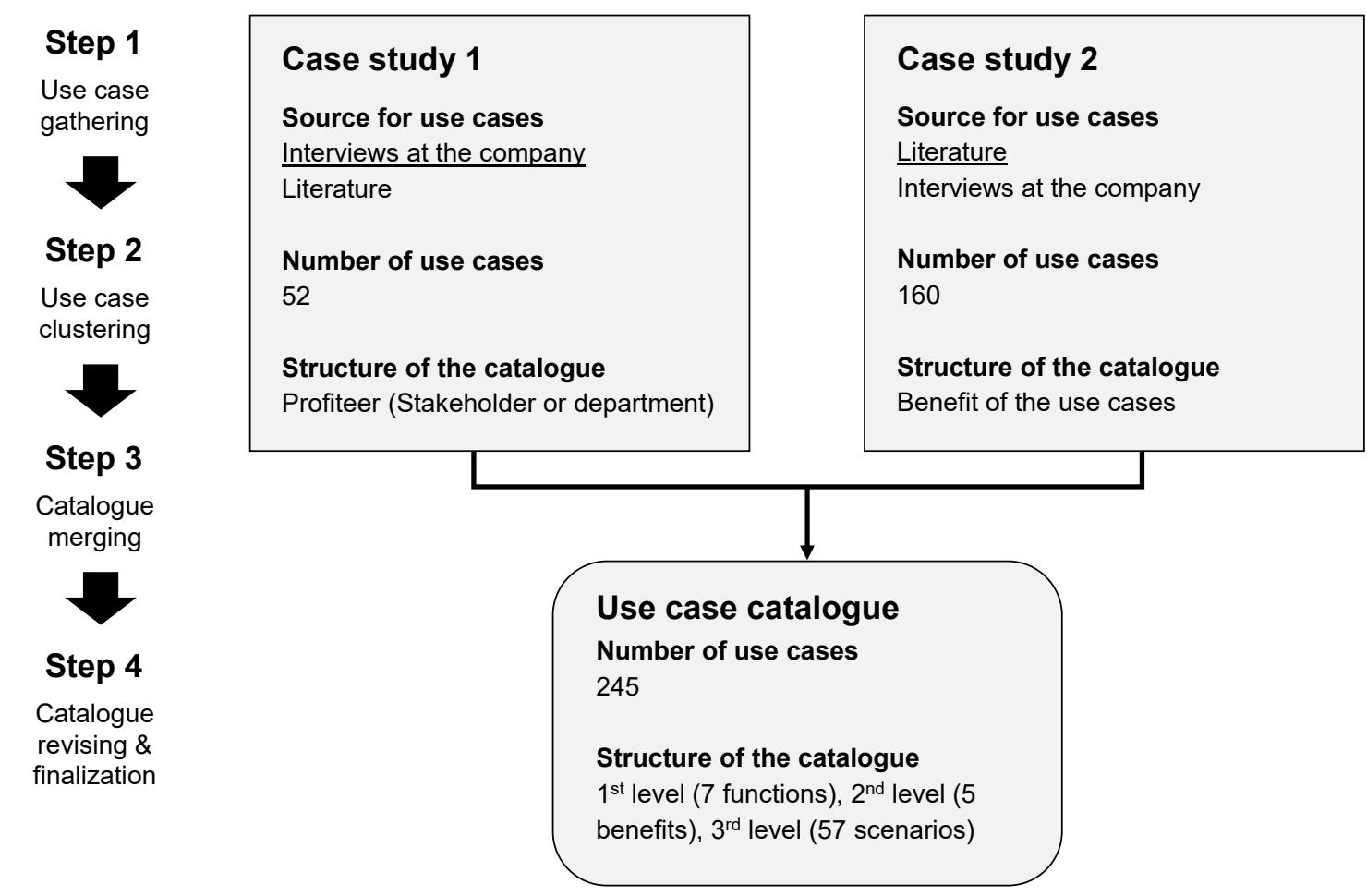

Figure 1. Research methodology leading to the use case catalogoue for IoT

Based on the research objectives, the authors developed a multi-stage research methodology that uses different sources for use cases. Figure 1 summarizes the developed research methodology that provided the input for the use case catalogue for IoT. In order to provide a comprehensive use case catalogue, the authors conducted two different case studies and analysed sources from literature to cover the broad range of use cases. However, due to the great complexity of this research field the authors decided to 
focus mainly on use cases that are linked to the management and development of the technical product and their related services (e.g., product development process, service engineering, or maintenance).

The underlying research approach of the use case catalogue is to use analogies to support companies in identifying new ideas regarding how to take advantage of use phase data provided by connected products. The research idea is to provide real example use cases or success stories from other companies and industries in a structured way so that the user of the catalogue can then transfer selected use cases into their own context. The reasoning is that using analogies can improve strategy development by increasing the efficiency of the process (Gavetti and Rivkin, 2005). Nevertheless, despite the benefits, analogies can also mislead decision makers and therefore their correct application is important. Thus, the use case catalogue will therefore also require a process for its application (See Section 4.1).

In order to derive a use case catalogue that provides value for research and industry, the authors used different sources to identify use cases. Two parallel case studies provided the foundation for the final use case catalogue. Both case studies lasted 6 months and were conducted at two different companies (one well-established mechanical engineering company with approximately 5,000 employees and one start-up from the medical equipment sector with less than 10 employees). The authors followed a multi case study approach to ensure that the use case catalogue is applicable in different industries (Eisenhardt, 1989). Furthermore, each student that was responsible for a case study conducted a literature review during the first step. For the literature review, the following sources served as input for use cases: journal papers, conference papers, reports, white papers, and news articles. Interviews with different stakeholders from various departments at the companies complemented the use case identification. During step 2, the authors tested two separate ways of clustering use cases. The first case study grouped the use cases based on the department that was the main profiteer (e.g., R\&D or service). The second case study grouped the use cases based on the benefit provided by each use case. However, it became clear that functional clustering helps companies to search for suitable use cases (see Section 4.2).

After completing both case studies, the two use case catalogues were compared and merged in order to derive one comprehensive use case catalogue. It became clear that paragraphs describing a use case occasionally contained multiple use cases. Therefore, use cases were further subdivided. The fourth step was to revise the entire catalogue. For this task, two three-hour long workshops helped to review and adjust the structure as well as content of the use case catalogue.

\section{Introduction of the use case catalogue for IoT}

\subsection{Application of the use case catalogue}

The research objective is to develop a tool that enables companies to identify novel use cases during their work on a use phase data strategy. The use case catalogue adopts the concept of analogy building to stimulate the ideation process. To avoid that the use case catalogue leads companies towards wrong use cases, it is important to understand the application process of such a tool. Research results show that analogy building can trigger innovative product ideas (Schild et al., 2005) or business models (Rumble and Minto, 2017). The assumption is that exploring the IoT use cases of other companies or industries that are provided by the catalogue helps to overcome thinking barriers and to trigger new ideas.

In general, using analogies entails that a person maps their own problems (target problem) to an external problem (source problem) (Gavetti and Rivkin, 2005). Afterwards, the external solution for the problem is transformed into their own personal solution. The risk is that a solution is transferred that does not fit the target problem because the source problem was not understood correctly. Figure 2 illustrates the application process for the use case catalogue. The starting point is a company that misses ideas for use cases for IoT during the strategy development because of various barriers (e.g., lack of experience with data or deadlocked ideas). Abstraction then helps to identify the core of the source problem (e.g., increase product's efficiency or guide user) and afterwards, analogy building allows for the development of suitable ideas for use cases. The developed catalogue provides concrete use cases (e.g., behaviour based payment for insurance or enhanced product functionalities via remote updates). The user takes those use cases as inspiration and selects interesting ones for their own business. The last step is to tailor the selected use case to the individual context. In this way, the use case catalogue helps to create novel ideas that serve as an input for the following steps of the strategy development. 
Gavetti and Rivkin (2005) mention four steps that help to avoid misleading analogy building: recognize the analogy and identify its purpose, understand the source, assess similarity, and translate, decide and adapt. Accordingly, the use case catalogue must provide use cases in a structured way so that users can assess if a selected use case suits the source problem. An understanding of the starting points for analogy building is very important because users might apply the catalogue under different circumstances. The authors identified four ways in which someone could start searching. The first option is to search for a use case based on the department in which the use case is applied (e.g., product development). The second option for the user is to directly search for a benefit that is provided by the use case for internal and external stakeholders (e.g., downtime reduction). Thirdly, a company might already have use phase data and therefore may want to know which other use cases they could implement with it (e.g., location data). Lastly, users can search for certain use cases titles directly (e.g., predictive maintenance).

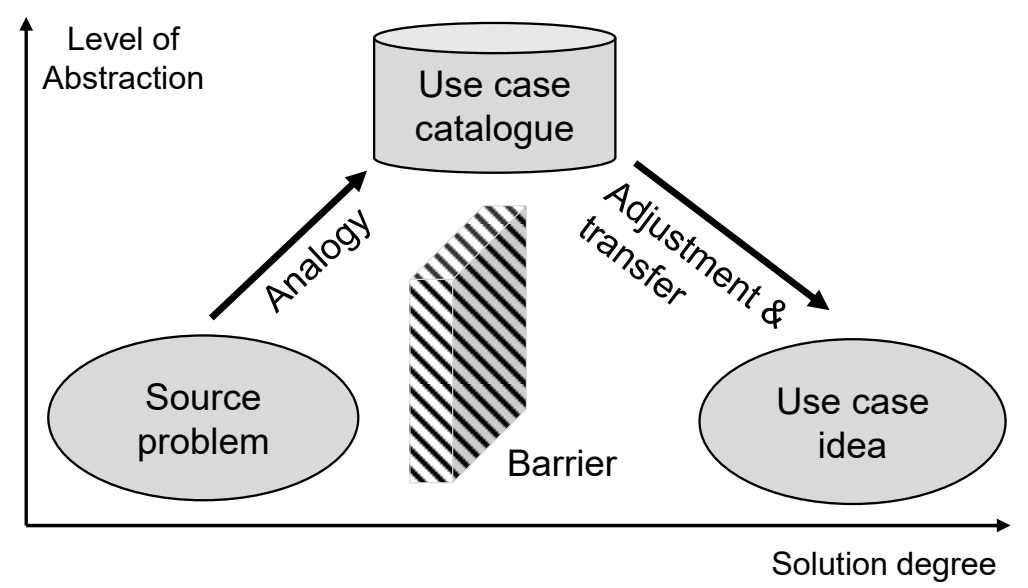

Figure 2. Application process for the use case catalogue

\subsection{Structure of the use case catalogue}

Prior to developing the final use case catalogue, two different options to cluster use cases were tested. However, it became clear that it is not ideal to cluster use cases only based on the department in which they were implemented because it bears the risk that users only search within the field of their target domain. On the other hand, searching use cases based only on their benefits did not appear suitable either because users might not be aware of the detailed benefit they want to achieve. Therefore, the authors decided to use three levels to cluster the more than 200 use cases. The different levels also respect the different entry points that companies may use when searching for a suitable use case that enriches the use phase data strategy. The first level divides use cases based on seven organizational functions of a manufacturing company (e.g., research and development, or service and product deployment). A comparison of the collected use cases highlighted that connectivity had mainly five positive benefits: performance improvement, stabilization/retention, individualization, monitoring \& analysis, and communication. Therefore, the second level separates use cases based on those five benefits. Using these different benefits across the organisational functions allows for the identification of use cases rooted in other departments that might be worthwhile to consider. The third level groups the use cases based on a broader application scenario that a use case belongs to. Figure 3 illustrates the structure of the use case catalogue. The left side shows the first two levels used to cluster the use cases. The right part shows how the performance improvement (second level) cluster is further separated into three application scenarios (third level), which describe the benefit a use case aims to provide. In total, 57 groups of application scenarios exist within the use case catalogue. However, it is also important to mention that one use case is not exclusively assigned to one sub-cluster (benefit) because use cases often provide multiple benefits and therefore a 1:1 relationship does not reflect the reality. The final catalogue was derived from two separate catalogues and, while merging both catalogues duplicates were removed and information provided for each use case was harmonised. 


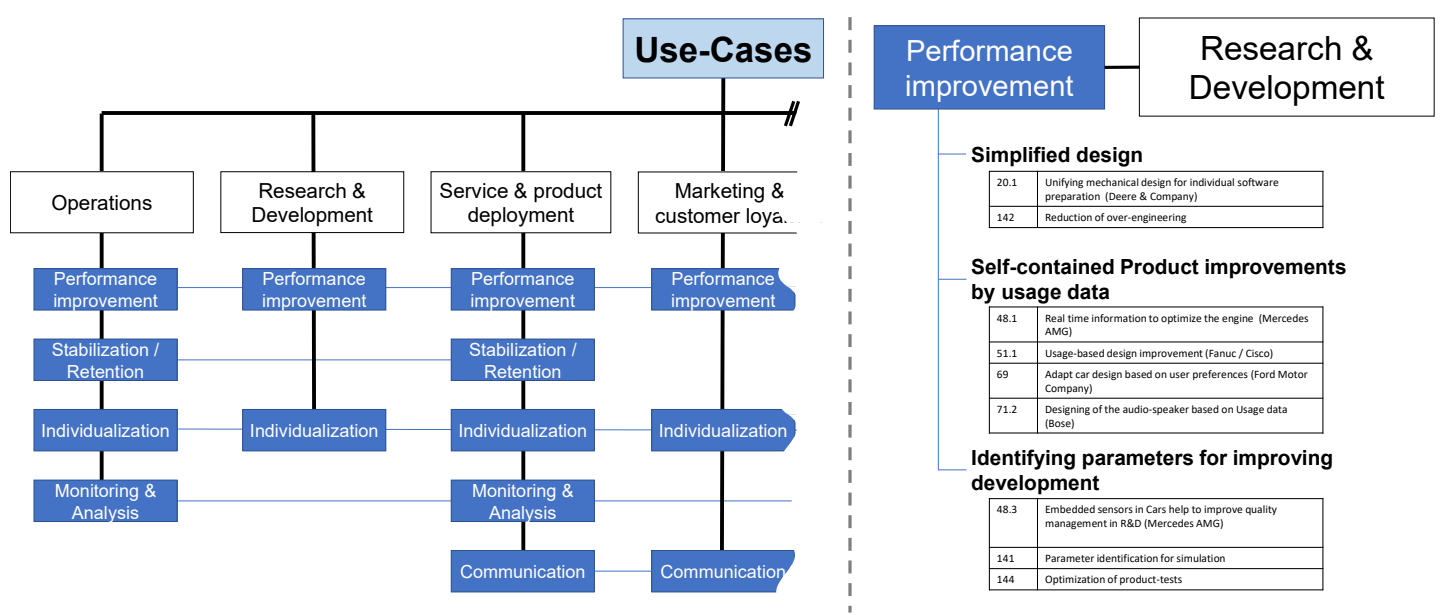

Figure 3. Excerpt of the structure of the developed use case catalogue

\subsection{Detailed description of the use case template}

The previous discussion on analogy building revealed that the main risk for users is drawing artificial analogies between the source and target problem. Therefore, the use case catalogue must provide sufficient information in order to enable the user to understand and pick suitable use cases. Besides the structure of the use case catalogue, the individual description of each use case is critical. The aspects describing each use case were derived based on information available in literature and experience gained during the case studies when discussing possible use cases. Discussions with practitioners showed that a clear textual description is important to specify the underlying process of a use case and the intended benefits in order to assess the suitability for the user's context. In addition, technical information for each use case helps to evaluate the effort for an implementation. Therefore, each use case within the catalogue is described with the following information:

- Project description - A few sentences describing the use case

- Use case name - Short name given to each use case

- Classification - Assignment to the three levels

- Cluster benefit - Short description of the value provided

- Applying company - Company and industry background for each use case

- IoT enabler - Technology enabling the use case (e.g., data analytics, AI, or real time data)

- Source of the use case - Reference to paper or additional documents

However, these categories only provide a very textual description of a use case whereas selecting a use case also requires the consideration of technical topics. Therefore, the authors decided to provide further information for each use case that is important for its implementation:

- Data type: location, condition, availability, or usage (Ellen MacArthur Foundation, 2016)

- Data analytics approach: descriptive, diagnostics, predictive, or prescriptive (Erwin et al., 2015)

- IoT functionality: monitoring, control, optimization, or autonomy (Porter and Heppelmann, 2014)

- Trigger for data transfer: event, interval, or continuous

The developed use case catalogue does not only provide a broad spectrum of use cases but also a very detailed description of each use case. Based on the needs of the user, the catalogue helps to derive ideas for possible use cases and in addition provides relevant details users might need to evaluate use cases or to plan their implementation. The developed catalogue enables all of these because users can explore options based on three levels and search based on their circumstances (e.g., available use phase data).

\subsection{Developed software support}

Originally, an Excel file contained all collected use cases and related information within the use case template, but it became clear that the usability of such a large and complex Excel file does not provide 
a user-friendly way to search for use cases. From the authors' perspective, a user wants to navigate easily through the catalogue and find use cases based on use case titles, benefits, or available use phase data (See Section 4.1). In addition, due to the large amount of information given for each use case, the concern was that the user might get put off by an Excel file with more than 20 columns. Thus, the objective was to provide all stored details only for use cases that the user selects. Therefore, the authors decided to develop a software prototype that makes the handling of the use case catalogue more userfriendly and intuitive. Figure 4 provides two screenshots of the software prototype, which show the two main windows of the tool that was programmed in C\# using the .net Framework.

A CSV file serves as an input for the software and then converts the file into an xml library. Therefore, users can use their own use case catalogue as an input as long as the data is provided in the right format. Furthermore, the software prototype has multiple options to search for suitable use cases. Users can search for a use case based on the provided structure (see Section 4.2), keywords, data features (e.g., type of use phase data or data analytics approach), and benefits (see Section 4.1).

The right part of Figure 4 illustrates an exemplary description of the use case "Ensure product quality based on data linkage". The figures show the example use case description, the assignment to the three levels of the catalogue and the other information described by the template (see Section 4.3). Due to the fact that users might need additional information for a use case, the software allows the user to edit each use case. Furthermore, the user can create a new use case in order to keep the catalogue up to date or to incorporate own their ideas. If different departments or stakeholders collaborate, the user is able to export the developed use case catalogue and share it with others using a CSV file.

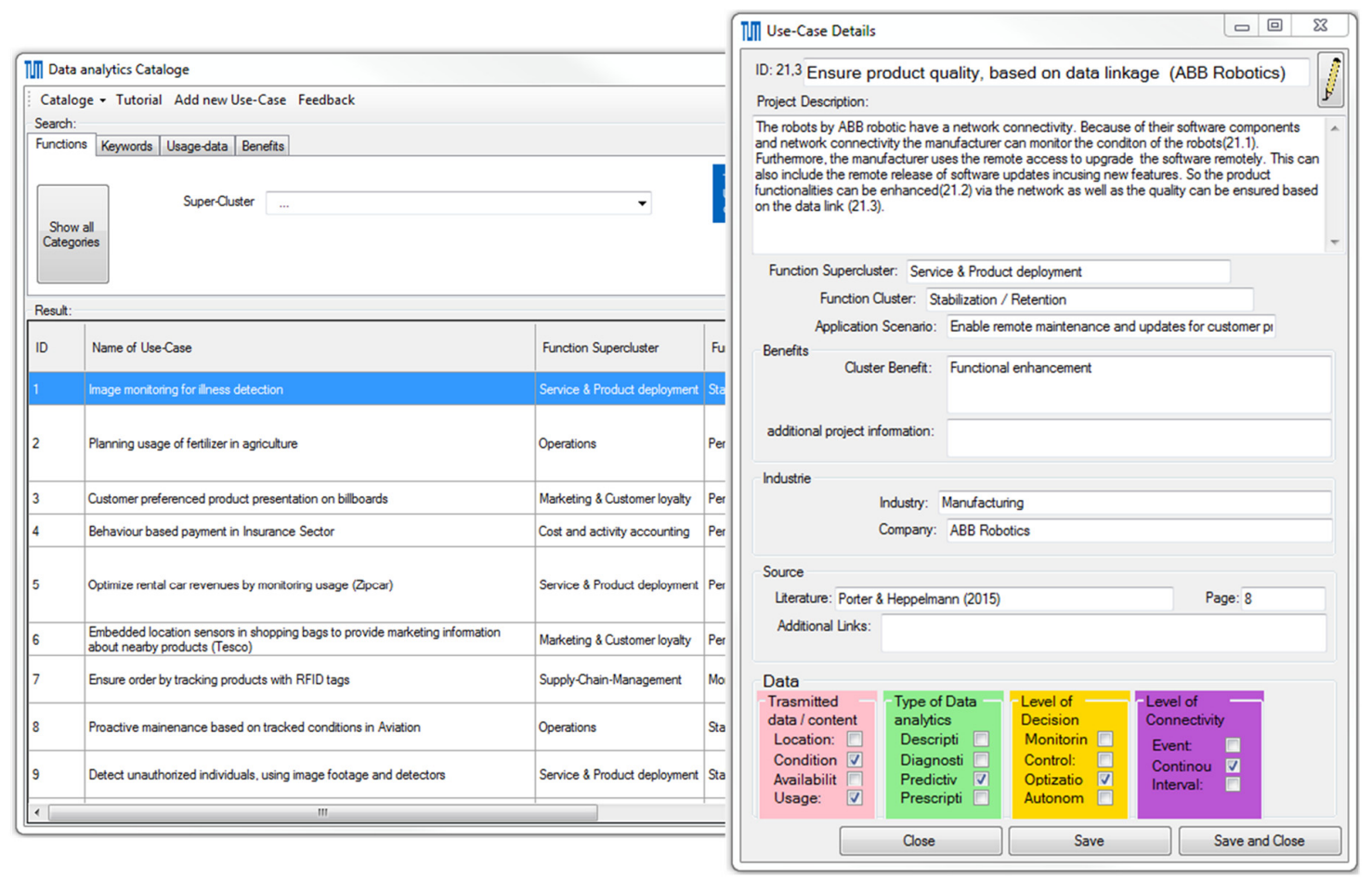

Figure 4. Screenshots of the developed software prototype for the use case catalogue

\section{Initial application of the use case catalogue during case studies}

In order to gain insights concerning the applicability, usability and usefulness of the use case catalogue, the authors conducted two case studies (each 6 months) in cooperation with engineering companies in order to obtain initial feedback. It is important to mention that these two case studies only provide a preliminary evaluation. The first application of the use case catalogue was part of the second case study (mentioned in the research methodology) during which the catalogue was initially developed. The second application case study was not part of the catalogue development itself and was conducted after 
the final catalogue was derived. The overall objective of the second application case was to develop a use phase data strategy for the company using the software tool during the search for suitable use cases. The first evaluation case study partner was a start-up from the medical equipment industry. The second company is a well-established manufacturer of dosing systems.

The objective of the first case study was to obtain initial feedback concerning the usefulness of the use case catalogue. A key aspect was to check the structure and comprehensiveness of the use case catalogue. In order to do this, a workshop was conducted together with the company that focused on the identification of possible use cases. The outcome was a collection of 29 potential use cases ( 21 requiring connectivity and 8 focusing on data analytics). The next step was then to compare the 21 IoT use cases with the use cases listed in the catalogue. The comparison revealed that the workshop led to four new use cases that were not mentioned in the catalogue and therefore two new use case clusters were added. Afterwards, an evaluation workshop was conducted that focused on the structure of the catalogue and its usefulness. The two interviewees got a demonstration of the catalogue and were able to use it. The feedback indicates that the structure and comprehensibility were very good. Furthermore, the participants mentioned that the use case catalogue is very useful to trigger the ideation of use cases and to illustrate the broad spectrum of IoT related use cases for the company.

The second application case study used the advanced version of the two use cases derived during the first two case studies. The objective of the application case study was to employ the catalogue during the development of a use phase data strategy for the mechanical engineering company. Over the course of the strategy development, 42 suitable use cases were derived. Out of those use cases, 11 use cases originated from using the catalogue. The main source for use case ideas (18 out of 42) was expert knowledge and individual ideas. The results show that the catalogue is a useful supplement during the search for novel use cases. However it also became clear that workshops, as well as the knowledge and experience of employees, are essential in order to derive a comprehensive list of use cases. During the second case study, competitive analysis also helped to identify additional use cases.

\section{Summary and outlook}

An increasing number of technical products get equipped with connectivity which enables them to communicate with other products or to transmit use phase data back to the manufacturer. Connectivity enables mechanical engineering companies to develop new products and related services that address user needs in a novel way. In addition, use phase data can also help to support internal stakeholders (e.g., engineering or service) by reducing over-engineering or improving the understanding of product failure. Different literature sources describe various use cases for IoT. However, these different sources appear as multiple puzzle pieces that are currently not connected to an overall picture. The literature review revealed that companies struggle especially with identifying suitable use cases. Developing a sound and successful use phase data strategy requires companies to explore the range of possible use cases. However, companies such as mechanical engineering companies that are especially inexperienced with offering connected products require additional support to identify suitable use cases for IoT.

Therefore, the research motivation was to help companies to overcome the barriers that they face when searching for novel ways to turn use phase data into value (e.g., new services or product features). Based on the insights gained from other research fields (e.g., business model development), the authors decided to develop a use case catalogue based on a literature review and two case studies in order to combine the research and the industry perspective. The idea behind the use case catalogue was to apply analogy building to support companies during the ideation phase. The use case catalogue provides use cases that describe how other companies turn use phase data into value, and this provides inspiration to derive use cases that fit to the user's company. In order to develop a broad use case catalogue, two case studies conducted independent literature reviews and interviews with employees at each case study partner. Afterwards, both catalogues were merged into one comprehensive use case catalogue that contains 245 use cases. Nevertheless, merely providing a long list of use cases did not seem very user friendly or useful. Therefore, the authors derived a structure for the use cases that clusters them on the following three different levels: organizational function (first level), desired benefit (second level), and application scenario (third level). In addition, the authors developed a software tool that allows the user to search the use case catalogue in different ways. Two case studies subsequently applied the use case catalogue 
to obtain initial feedback from industry. The findings show that the catalogue provides inspiration for additional use cases that were not discussed before and therefore has a positive effect on the ideation phase during the development of a use phase data strategy. However, the case studies also emphasized that companies should use the catalogue in combination with internal workshops and a competitive analysis. Overall, the developed use case catalogue appears to be a useful tool that helps companies to identify additional use cases that help them to take advantage of their connected products and services. Even though the initial feedback seems promising, additional research is still needed to further improve the use case catalogue. The first research task should be a more structured evaluation, which is already planned. The objective should be to measure the real added value of the catalogue by comparing the outcome of two teams (one with the catalogue and one without). Further insights would be helpful in order to understand how practitioners evaluate the usability and usefulness of the software tool. Currently the data analytics perspective is not integrated into the use case catalogue. A goal should be to provide a suggestion for each use case that points the user towards suitable algorithms. The challenge that will remain is that often different algorithmic solutions exist. A main challenge during the development of the use case catalogue was that literature sources often described use cases on different abstraction levels. Thus, the authors are currently aligning the information provided for each use case.

\section{Acknowledgment}

We thank the German Research Foundation (Deutsche Forschungsgemeinschaft - DFG) for funding this project as part of the collaborative research center 'Sonderforschungsbereich 768 - Managing cycles in innovation processes - Integrated development of product-service systems based on technical products'.

\section{References}

Alharthi, A., Krotov, V. and Bowman, M. (2017), “Addressing barriers to big data”, Business Horizons, Vol. 60 No. 3, pp. 285-292. https://doi.org/10.1016/j.bushor.2017.01.002

Bange, C., Grosser, T. and Janoschek, N. (2015), "Big data use cases. Getting real on data monetization", Business Application Research Center (BARC).

Barton, D. and Court, D. (2012), "Making advanced analytics work for you”, Harvard Business Review, Vol. 90 No. 10, pp. 78-83.

Berman, S.J. and Bell, R. (2011), "Digital transformation. Creating new business models where digital meets physical”, IBM Institute for Business Value, pp. 1-17.

Bloching, B., Leutiger, P., Oltmanns, T., Rossbach, C., Schlick, T. et al. (2015), Die digitale Transformation der Industrie, Roland Berger Strategy Consultants GmbH \& Bundesverband der Deutschen Industrie, München.

Capgemini and EMC (2015), Big \& Fast Data: The Rise of Insight-Driven Business - Engineering.

Carnero, M.C. (2005), "Selection of diagnostic techniques and instrumentation in a predictive maintenance program. A case study", Decision Support Systems, Vol. 38 No.4, pp. 539-555. https://doi.org/10.1016/j.dss.2003.09.003

Chui, M., Löffler, M. and Roberts, R. (2010), “The Internet of Things”, McKinsey Quarterly.

Davenport, T. (2014), Big data at work: dispelling the myths, uncovering the opportunities // Big Data at Work: Dispelling the Myths, Uncovering the Opportunities, Harvard Business Review Press, Boston.

Deldin, J.-M. and Schuknecht, M. (2014), "The AskNature Database. Enabling Solutions in Biomimetic Design", In: Goel, A.K., McAdams, D.A. and Stone, R.B. (Eds.), Biologically Inspired Design: Computational Methods and Tools, Springer London, London, pp. 17-27. https://doi.org/10.1007/978-1-4471-5248-4_2

Demchenko, Y., de Laat, C. and Membrey, P. (2014), "Defining architecture components of the Big Data Ecosystem", International Conference on Collaboration Technologies and Systems (CTS).

Dinter, B., Franz, T., Grapenthin, S., Konrad, R., Nienke, S. et al. (2015), Big Data und GeschäftsmodellInnovationen in der Praxis: 40+ Beispiele, BITKOM, Berlin.

Dutta, D. and Bose, I. (2015), "Managing a big data project: the case of ramco cements limited", International Journal of Production Economics, Vol. 165, pp. 293-306. https://doi.org/10.1016/j.ijpe.2014.12.032

Eisenhardt, K.M. (1989), "Building theories from case study research", Academy of management review, Vol. 14 No. 4, pp. 532-550.

Ellen MacArthur Foundation (2016), Intelligent Assets: Unlocking the Circular Economy Potential.

Erwin, T., Heidkamp, P. and Pols, A. (2015), Mit Daten Werte schaffen, Bitcom Research GmbH.

Fitzgerald, M., Kruschwitz, N., Bonnet, D. and Welch, M. (2014), "Embracing digital technology. A new strategic imperative", MIT sloan management review, Vol. 55 No. 2, p. 1. 
Fleisch, E., Weinberger, M. and Wortmann, F. (2017), “Geschäftsmodelle im Internet der Dinge”, In: Reinheimer, S. (Ed.), Industrie 4.0: Herausforderungen, Konzepte und Praxisbeispiele, Springer Fachmedien Wiesbaden, Wiesbaden, pp. 1-16. https://doi.org/10.1007/978-3-658-18165-9_1

Gassmann, O., Frankenberger, K. and Csik, M. (2014), The business model navigator: 55 models that will revolutionise your business, Pearson, Harlow.

Gavetti, G. and Rivkin, J.W. (2005), "How strategists really think”, Harvard Business Review, Vol. 83 No. 4, pp. 54-63.

Henke, N., Bughin, J., Chui, M., Manyika, J., Saleh, T. et al. (2016), The age of analytics: Competing in a datadriven world, McKinsey Global Institute.

Kaeser, J. (2017), "From Data to Business. A Paradigm Shift in Industry”, Evolving Business Models, Springer, pp. 141-152.

Kusiak, A. and Smith, M. (2007), "Data mining in design of products and production systems", Annual Reviews in Control, Vol. 31 No. 1, pp. 147-156.

LaValle, S., Lesser, E., Shockley, R., Hopkins, M.S. and Kruschwitz, N. (2011), "Big data, analytics and the path from insights to value", MIT sloan management review, Vol. 52 No. 2, p. 21.

Muhtaroğlu, F.C.P., Demir, S., Obalı, M. and Girgin, C. (2013), "Business model canvas perspective on big data applications", 2013 IEEE International Conference on Big Data, pp. 32-37.

O’Halloran, D., Kvochko, E., Daugherty, P., Reilly, M., Banerjee, P. et al. (2015), "Industrial internet of things unleashing the potential of connected products and services", World Economic Forum.

Porter, M.E. and Heppelmann, J.E. (2014), "How smart, connected products are transforming competition", Harvard Business Review, Vol. 92 No. 11, pp. 64-88.

Porter, M.E. and Heppelmann, J.E. (2015), "How smart, connected products are transforming companies", Harvard Business Review, Vol. 93 No. 10, pp. 53-71.

Rapolu, B. (2016), Internet Of Aircraft Things: An Industry Set To Be Transformed. [online] Available at: $\mathrm{http}$ //aviationweek.com/connected-aerospace/internet-aircraft-things-industry-set-be-transformed (accessed 26.11.2017).

Rumble, R. and Minto, N.A. (2017), "How to use analogies for creative business modelling", Journal of Business Strategy, Vol. 38 No. 2, pp. 76-82. https://doi.org/10.1108/JBS-09-2016-0091

Schild, K., Herstatt, C. and Lüthje, C. (2005), "How to use analogies for breakthrough innovations", Working Paper, Vol. 24, Universitätsbibliothek der Technischen Universität Hamburg-Harburg, Hamburg-Harburg.

Schmidt, D.M., Jaugstetter, M., Malaschewski, O. and Mörtl, M. (2015), "Service Classification to Support Planning Product-Service Systems”, Asian Design Engineering Workshop (A-DEWS), Hong Kong.

van der Vegte, W.F. (2016), "Taking Advantage of Data Generated by Products: Trends, Opportunities and Challenges", ASME 2016 International Design Engineering Technical Conferences and Computers and Information in Engineering Conference, Charlotte, USA, August 21-24, 2016.

Wilberg, J., Schäfer, F., Kandlbinder, P., Hollauer, C., Omer, M. and Lindemann, U. (2017a), "Data Analytics in Product Development: Implications from Expert Interviews", 2017 IEEE International Conference on Industrial Engineering and Engineering Management (IEEM), Singapore, December 10-13, 2017.

Wilberg, J., Triep, I., Hollauer, C. and Omer, M. (2017b), "Big Data in product development: Need for a data strategy", Proceedings of the PICMET '17: Technology Management for Interconnected World, Portland, USA. https://doi.org/10.23919/PICMET.2017.8125460

Wirth, N. and Wirth, R. (2017), "How to meet the four key challenges to turn Big Data into smart data-driven solutions", Research World, Vol. 2017 No. 64, pp. 31-36.

Xia, F., Yang, L.T., Wang, L. and Vinel, A. (2012), "Internet of things", International Journal of Communication Systems, Vol. 25 No. 9, pp. 1101-1102.

Julian Wilberg, Research Associate

Technical University of Munich, Product Development and Lightweight Design

Boltzmannstr. 15, 85748 Garching, Germany

Email: wilberg@pl.mw.tum.de 